\title{
Reconstructing a Performance by Johannes Brahms
}

\author{
James Rosser \\ Email: james.rosser10@gmail.com
}

\begin{abstract}
The 1889 wax cylinder recording of Brahms's Hungarian Dance No. 1, performed by the composer, is a fascinating piece of music history. However, today the cylinder is in very poor physical condition and the musical content of surviving copies became almost inaudible before it could be digitised. The cylinder attracted academic interest but conclusions that Brahms used underdotting and improvisation in his performance have made a musically satisfying reconstruction seem unlikely. This paper challenges these conclusions by interpreting note timings from the recording in the context of recent advances in knowledge of past performance practices. The result is an interpretation which remains faithful to the original score and can be performed in a musically satisfying manner, affording insight into Brahms and piano playing in general.
\end{abstract}

\section{INTRODUCTION}

The 1889 wax cylinder recording of Brahms's Hungarian Dance No. 1, performed by the composer, is a fascinating piece of music history. However, today the cylinder is in very poor physical condition, and the best sound quality is preserved on a 1935 transfer to gramophone disc undertaken by the Institute for Sound Research at the University of Berlin[1]. Acetates believed to be copies of this transfer were held by the British Library National Sound Archive and released by Desmar Records in 1977[2] and VÖAW in 1983[3]. These releases renewed academic interest in the recording but commentators struggled to reach definitive conclusions on the musical value of its contents. In 1994 Jonathan Berger and Charles Nichols produced a denoised version of the recording using wavelet packet signal analysis[4] and analysed the results in detail. Despite these efforts, the musical insight we would hope to gain from a performance by Brahms remains elusive.

However, recent advances in our understanding of performing practices in piano playing of the late 19th and early 20th centuries, as detailed in Neal Peres Da Costa's Off The Record (2012)[5], have made it possible to reinterpret the data from the cylinder through the lens of a long extinct style. As a result this paper proposes an interpretation of the recording which can be reconstructed at the piano in its entirety while remaining consistent with both the original score and the timings observed in the recording.

\section{Current State of AnAlysing the Brahms Cylinder}

In the liner notes for the Desmar 1977 release of the recording, Gregor Benko remarked that "any musical value ... can be charitably described as the product of a pathological imagination"[2]. However, Will Crutchfield's analysis in Opus (1986) makes numerous musical observations from the recording, claiming to detect tempo modification and dislocation[6]. Costa (2012) adds to this by commenting that "Brahms intended the lengthening of single notes as well as the broadening of particular bars ... while the speeding up of certain phrases certainly adds to their excitement"[5, p. 268]. The more scientific approach of Berger and Nichols (1994)[4] provided stronger evidence to support the theory of tempo modification. Regarding the bars which terminate the four-bar phrase groups in bars 49-68 they conclude: "These measures become significantly extended, with overall durations of 1.033 seconds for measure 52 and 1.146 seconds for measure 68 in contrast to the average duration of 806 milliseconds per measure"[4]. As a result of these efforts the recording from bar 49 is largely understood. All sources agree that Brahms largely follows the score but greatly modifies tempo, emphasising the Hungarian dance rhythm at the end of phrases with significant pauses.

However sources struggle to come to consensus on the preceding bars, with a number of problems undermining the leading explanations. Crutchfield (1986) claims to hear separation of the left and right hands on "accented first beats where the texture is melody/accompaniment"[6]. Costa (2012) supports this conclusion, stating that "Brahms can be heard dislocating melody from accompaniment quite regularly" [5, p. 76] but neither provide a scientifically informed case for dislocation. In bars 13-46 Berger and Nichols (1994) identify a "general tendency towards underdotting", observing that "Brahms gives the dotted quarter note its full value only once"[4]. However there is little historical evidence for underdotting as an expressive device and Joseph Joachim's 1903 recording of the same piece arranged for violin did not show a tendency for underdotting despite his close association with Brahms.

Berger and Nichols (1994) also propose that improvisation is present at a number of points in the recording, stating that added ornaments are present in bars 17 and 60 as well as "in measures 16, 20, 39, and 46"[4]. However they acknowledge that "detection of arpeggios and groups of notes with short durations proved problematic"[4] and I was unable to validate these observations by ear as registral extremes are very poorly captured on the recording. Of bars 25-36 they remark that "Brahms alters this group considerably in his performance"[4] and their transcription from the observed note timings bears little resemblance to the piece. Berger and Nichols acknowledged that "a musically pleasing ... reconstruction of this recording was not currently feasible"[4] but noted that it was of interest to provide "a glimpse of [Brahms] taking leave of the score in his own performance"[4]. Unfortunately such a disregard for the score seems troubling, especially as no witnesses to the event recorded any indication that Brahms was improvising. 


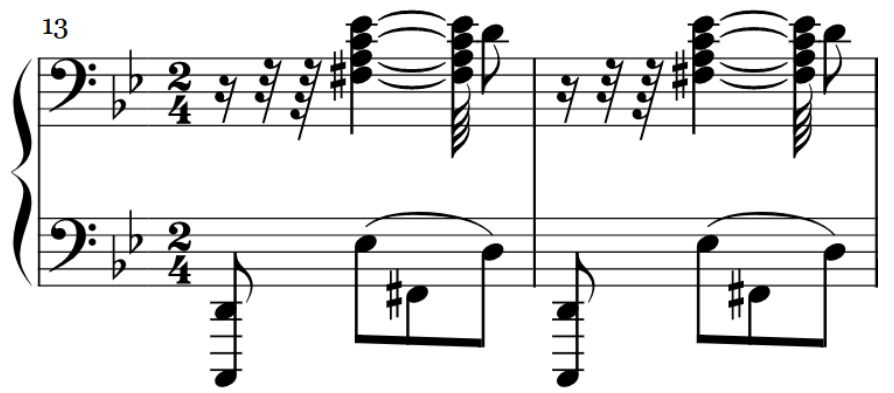

Fig. 1: Original score edited to show literal notation for dislocation

\section{NEW APPROACH}

\section{Dislocation}

Costa (2012) defines dislocation as "the practice of playing one hand separate from the other" and argues that it was commonplace in late 19th and early 20th century piano playing[5]. The poor quality of the recording inhibits ascertaining note durations and the bass and other accompanying parts are inaudible. In A Generative Theory of Tonal Music Lerdahl and Jackendoff (1996) discuss the perception of metrical structure and remark: "The bass tends to be metrically more stable than the upper parts: when it plays isolated notes, they are usually strong beats"'[7, p. 87]. The recording therefore does not contain sufficient information to infer metrical structure and as a result onset timings that signify underdotting can be reinterpreted as dislocation. To do so, we assume that the first melody note of each bar is preceded by an unheard bass note. The second melody note then occupies its usual place as the last quaver of the bar. Figure 1 presents a notated example.

In her recollections of Brahms, Fanny Davies remarked: "The sign " $<>$ ", as used by Brahms ... applied not only to tone but to rhythm also"[8]. Abundant use of hairpins in bars 13-24 along with espress markings in bars 1, 7 and 13 lends further support to the use of dislocation[9].

\section{Improvisation}

Berger and Nichols (1994) produced a transcription of bars 25-36 suggesting substantial improvisation in the form of both "distortion of the metric layout" and "prominent melodic insertion within the phrase structure of the original score" but they acknowledge that they "have yet to isolate the accompaniment and all the internal detail of this phrase"[4]. As mentioned above, the lack of accompanying bass parts leaves little merit in inferring metrical structure from what is audible on the recording. Figure 2 presents the note values from this transcription[4] on the upper stave, with barlines shifted one quaver to the right. The lower two staves show a simplified version of the original piano part with cross notes added to aid comparison. It can easily be seen that the first passage matches almost exactly and the second passage matches with the exception of some lost time in the third bar. This demonstration severely undermines the conclusion that Brahms improvised this section.

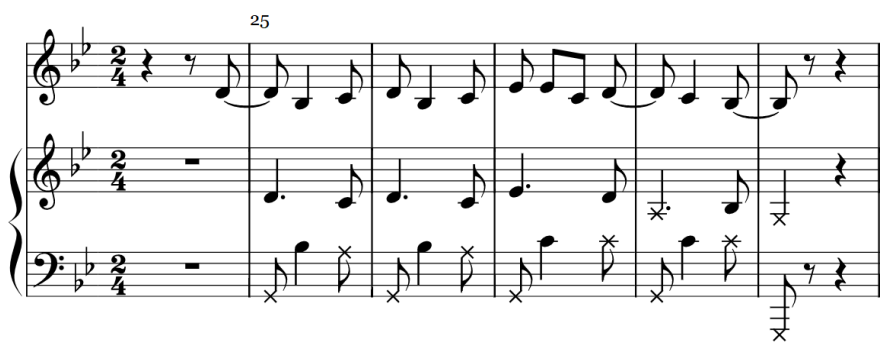

(a) Phrase 1

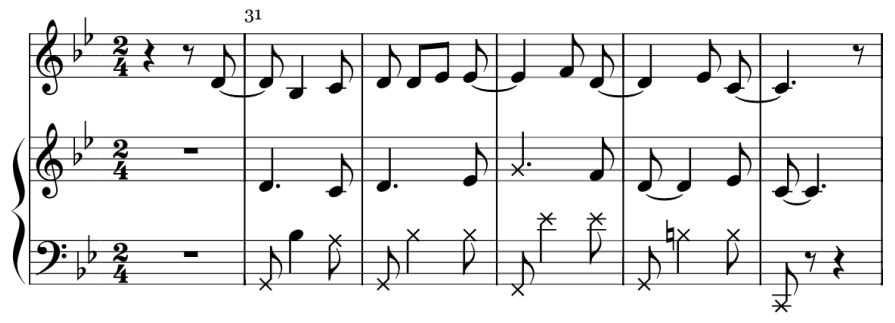

(b) Phrase 2

Fig. 2: Note lengths from the Brahms at the Piano[4] Transcription for bars 25-35 (upper stave) compared with the simplified original score (lower two staves) with cross notes indicating the surrounding parts to aid comparison

I completed a new analysis of note timings in bars 25-48 by stepping through the denoised recording produced by Berger and Nichols (1994)[4] in a digital audio editor and labelling onset times by ear for each clearly audible note. The results are shown in Tables I and II. Duration for a note was calculated as the difference between its onset and the onset of the note following. Onset times for three notes at the start of bars couldn't be determined. These were estimated by assuming the length of the preceding note from previous occurrences of the same rhythmic pattern. For example to estimate onset for the D that starts bar 39 the quaver D in bar 38 was assigned the same duration as the quaver D in bar 37. For validation, durations were compared to timings from Berger and Nichols[4] and found to match very closely.

To produce the transcriptions in Figure 3, the length of each bar was calculated by taking the duration between the first note of a bar and the first note of the bar following. Note values were then assigned based on their duration as a proportion of the length of the bar. Estimated notes are shown as cross notes.

Comparing recordings of pianists from 1903-1992, Costa (2012) notes that "the earlier pianists generally make larger and therefore more noticeable changes [in tempo] from bar to bar' [5, p. 252] and presents data which shows examples of La Forge, Pachmann and Rosenthal doubling tempo between bars. Of a detailed account by Fanny Davies regarding tempo changes during Brahms's performance of the 4th movement of his Piano Trio Op. 101, Costa remarks: "Her figures show a wide variation of tempo that is not evident from Brahms's indications alone" and that "the term poco stringendo results in a variation from $76 \mathrm{MM}$ to $120 \mathrm{MM}$ "[5, p. 264]. 
TABLE I: Observed note timings in seconds for bars 25-36 compared with timings from Brahms at the Piano[4]

\begin{tabular}{|c|c|c|c|c|}
\hline Note & Onset & Duration & BATP Onset & BATP Duration \\
\hline D & 0 & 0.692 & 0 & 0.992 \\
\hline C & 0.692 & 0.249 & 0.992 & 0.241 \\
\hline D & 0.941 & 0.487 & 1.233 & 0.604 \\
\hline C & 1.428 & 0.203 & 1.837 & 0.188 \\
\hline E & 1.631 & 0.312 & 2.025 & 0.313 \\
\hline C & 1.943 & 0.321 & 2.338 & 0.346 \\
\hline D & 2.264 & 0.217 & 2.684 & \\
\hline A & 2.481 & 0.099 & & \\
\hline C & 2.58 & 0.447 & 3.008 & 0.431 \\
\hline B b & 3.027 & 0.428 & 3.439 & \\
\hline G & 3.455 & & & \\
\hline \hline D & 5.155 & 0.596 & 5.556 & 0.643 \\
\hline C & 5.751 & 0.259 & 6.199 & 0.246 \\
\hline D & 6.01 & 0.313 & 6.445 & 0.317 \\
\hline Eb & 6.323 & 0.3 & 6.762 & \\
\hline G & 6.623 & 0.572 & & \\
\hline F & 7.195 & 0.23 & 7.511 & 0.306 \\
\hline D & 7.425 & 0.497 & 7.817 & 0.526 \\
\hline Eb & 7.922 & 0.303 & 8.343 & 0.314 \\
\hline C & 8.225 & & 8.657 & \\
\hline & & & & \\
\hline
\end{tabular}

TABLE II: Observed note timings in seconds for bars 37-48

\begin{tabular}{|c|c|c|}
\hline Note & Onset & Duration \\
\hline $\mathrm{E} b$ & 9.882 & 0.657 \\
\hline $\mathrm{D}$ & 10.539 & 0.3 \\
\hline $\mathrm{E} b$ & 10.839 & 0.561 \\
\hline $\mathrm{D}$ & 11.4 & $(0.3)$ \\
\hline Estimated D & 11.7 & 0.344 \\
\hline $\mathrm{C}$ & 12.044 & 0.323 \\
\hline $\mathrm{A}$ & 12.367 & 0.476 \\
\hline $\mathrm{B} b$ & 12.843 & 0.422 \\
\hline $\mathrm{A}$ & 13.265 & \\
\hline \hline $\mathrm{E} b$ & 14.934 & 0.618 \\
\hline $\mathrm{D}$ & 15.552 & 0.332 \\
\hline $\mathrm{E} b$ & 15.884 & 0.532 \\
\hline $\mathrm{D}$ & 16.416 & $(0.332)$ \\
\hline Estimated D & 16.748 & 0.438 \\
\hline $\mathrm{A}$ & 17.186 & 0.181 \\
\hline $\mathrm{C}$ & 17.367 & 0.596 \\
\hline $\mathrm{B} b$ & 17.963 & $(0.332)$ \\
\hline Estimated G & 18.295 & \\
\hline
\end{tabular}

The $\mathrm{C}$ in bar 28 arrives 0.1 seconds after the preceding A compared to the typical quaver duration of 0.25 seconds. Interpreting this unusually early arrival as a bar played at suddenly increased tempo, and the delayed onset of the $\mathrm{G}$ in bar 29 as dislocation, provides an explanation for the observed timings. The same techniques can be applied to bars $31-48$ as follows: Suddenly increased tempo is applied to bars 32,34 and 39 and dislocation of the first melody note is applied in bars 33, 35, 40 and 41. The only modification in Phrase 4 of this section is overdotting of the last note of bar 45 .

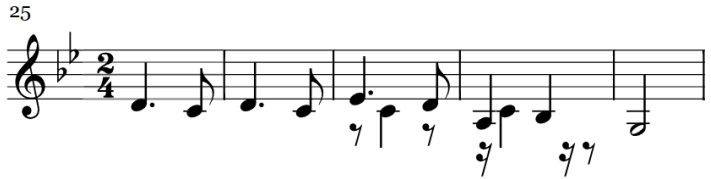

(a) Phrase 1

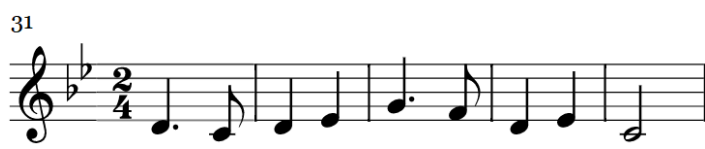

(b) Phrase 2

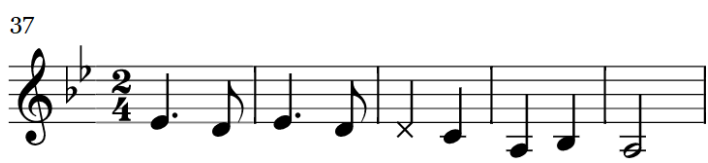

(c) Phrase 3

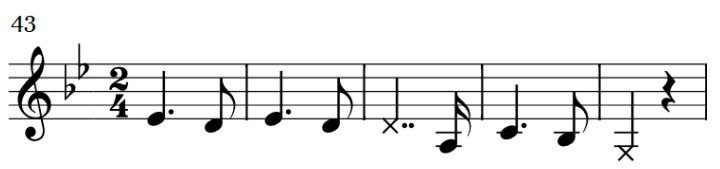

(d) Phrase 4

Fig. 3: Transcriptions for each phrase in bars 25-48 based on the timings in Tables I and II. Cross notes are used to signify notes which couldn't be detected in the recording.

Despite these distortions there is little doubt the listener would still recognise this passage from the score, the altered rhythms of the melody unable to override the influence of the accompaniment. The Hungarian dance rhythm is also preserved throughout. In addition, this analysis results in four distinct rhythmic transformations. The deliberate nature of these modifications seems strong evidence of an intentional stylistic decision by Brahms; a valuable musical insight from the 19th century not recorded in notation. As a result of experiments at the keyboard, it is the opinion of the author that these techniques produce a musically satisfying result.

\section{FURTHER ANALYSIS}

On listening to the extract played in full according to this interpretation, the result feels intuitively closer to the score than might be expected given the scale of alterations to the musical surface. In order to justify this intuition, rules described by Lerdahl and Jackendoff (1996) can be applied to demonstrate how transformation from surface features to the perceived result might take place[7]. A primary effect of dislocation is syncopation, described by Lerdahl and Jackendoff as taking place "where cues are strongly contradictory yet not strong enough, or regular enough, to override the inferred [rhythmic] pattern" [7, p. 17]. The perception of time signature continues to align with the score but contradictory signals increase ambiguity.

Lerdahl and Jackendoff (1996) also explore the concept of reductions, which arise from the observation that "some musical passages are heard as ornamented versions, or elaborations, of others" $[7, \mathrm{p} .105]$. To form a reduction they segment a piece into domains of elaboration known as time-spans, described as 
"apprehended rhythmic units in terms of which pitch structure is heard"'7, p.119,124]. One aspect of reduction is fusion, described as follows: "the head [of a time-span] may be the fusion of the events into a single event; or, conversely, the surface events may be an arpeggiation of the head"'[7, p. 153]. When applied to dislocation, this process of fusion would cause the listener to perceive the bass and dislocated chord together at a higher reductional level, thereby perceiving the structure notated in the score.

Tempo modification in bars $25-48$ causes main beats to occur at less regular time intervals. However, the first Metrical Preference Rule proposed by Lerdahl and Jackendoff states: "Where two or more groups or parts of groups can be construed as parallel, they preferably receive parallel metrical structure"[7, p. 75]. As each bar can be recognised as a familiar rhythmic structure the listener is able to perceive the structure of the original score from the musical surface. Curiously reminiscent of these observations is Fanny Davies's remark of Brahms's playing that "one felt the fundamental rhythms underlying the surface rhythms"[8].

There has long been a debate in music over how literal a composer should be with their instructions in the score. As shown in Figure 1, literal notation immediately becomes clumsy when trying to describe subtle rhythmic alterations. As successful use of dislocation requires balancing many contextual factors, even if timings could be notated precisely and clearly, there is no guarantee that the context would suit them in all cases. The observations by Berger and Nichols (1994) for bars 13-46 suggest that Brahms dislocates with a range of timings, from dislocating by a full quaver to not at all[4]. Precise notation of these subtleties would not be practical. Similar problems exist for tempo modification. Exact notation would involve tempo markings for individual bars, which would be very challenging for a performer to follow and inflexible to context. Costa (2012) provides a number of examples of commentators expressing the opinion that the score is inadequate for capturing the subtleties of expression, including the observation that "Although Chopin renounced the use of terminology, it is clear that he tried to notate metrical rubato alterations in his compositions. This met with severe opposition that highlighted the weakness inherent in the notation of such alterations"' 5 , p. 235]. As a result the musical context of a time period clearly plays a highly significant role in understanding a composer's intentions from the score.

\section{CONCLUSION}

This paper challenges the notions that Brahms used underdotting or improvisation in his 1889 recording, by proposing an alternative interpretation which is consistent with the observed timings. This interpretation remains faithful to the original score and only makes use of well documented techniques known to have been in use when the recording was made. The end result is musically satisfying and can be reconstructed in full, offering insight into Brahms as a performer as well as a performer's relationship to the score in piano music from the Romantic Era.

\section{REFERENCES}

[1] H. Kowar, F. Lechleitner, and D. Scholler, "Zur wiederherausgabe des einzigen tondokuments von johannes brahms durch das phonogrammarchiv," Schallarchiv, December 1983.

[2] G. Benko, "Landmarks of recorded pianism volume 1 - acoustic recordings (1889-1924)," LP, 1977.

[3] "Johannes brahms und sein freundeskreis - tondokumente aus dem phonogrammarchiv der österreichischen akademie der wissenschaften," PHA EP 5, 1983.

[4] J. Berger and C. Nichols, "Brahms at the piano: An analysis of data from the brahms cylinder," Leonardo Music Journal, vol. 4, p. 23, 1994.

[5] N. P. D. Costa, Off the record: performing practices in romantic piano playing. Oxford University Press, 2012.

[6] W. Crutchfield, "Brahms, by those who knew him," Opus, vol. 2, p. 14 August 1986.

[7] F. Lerdahl and R. Jackendoff, A Generative Theory of Tonal Music. MIT Press, 1996.

[8] F. Davies, "Some personal recollections of brahms as pianist and interpreter," in Cobbett's Cyclopedic Survey of Chamber Music, W. Cobbett and C. Mason, Eds. Oxford: Oxford University Press, 1963, vol. 1, pp. $182-4$.

[9] J. Brahms, Twenty One Hungarian Dances for Pianoforte. London: Lengnick, 1919. 